\title{
Vaccination adjuvated against hepatitis B in Spanish National Healthcare System (SNS) workers typed as non-responders to conventional vaccines
}

\author{
José L. Bravo-Grande ${ }^{\mathrm{a}, *}$, M. Asunción Blanco-González ${ }^{a}$, José M. de la Torre-Robles ${ }^{\mathrm{b}}$, \\ Marita P. Asmat-Inostrosa ${ }^{\mathrm{b}}$, Marina Fernández-Escribano ${ }^{\mathrm{c}}$, Itria Mirabella Villalobos ${ }^{\mathrm{c}}$, \\ M. Covadonga Caso-Pita ${ }^{\mathrm{d}}$, Marina Hervella-Ordoñez ${ }^{\text {, }}$ Luis M. Cañibano Cimas ${ }^{\text {e }}$, \\ José M. de la Fuente-Martín ${ }^{\mathrm{f}}$, M. Luisa Rodríguez de la Pinta ${ }^{\mathrm{g}}$, Jenry Ricardo Borda Olivas ${ }^{\mathrm{g}}$, \\ Carmen Muñóz-Ruipérez ${ }^{\mathrm{h}}$, Miguel A. Alonso López ${ }^{\mathrm{h}}$, MT del Campo ${ }^{\mathrm{i}}$, \\ M. Antonieta Ramírez Pérez ${ }^{i}$, Ignacio Sánchez-Arcilla ${ }^{j}$, Marco Marzola-Payares ${ }^{j}$, \\ Fernando Rescalvo-Santiago ${ }^{\mathrm{k}}$, Miguel de Paula-Ortiz ${ }^{\mathrm{k}}$, José M. Sánchez-Santos ${ }^{1}$, Ricardo López-Pérez ${ }^{\mathrm{m}, *}$ \\ a Servicio de Prevención de Riesgos Laborales, Complejo Asistencial Universitario de Salamanca, Salamanca, Spain \\ ${ }^{\mathrm{b}}$ Servicio de Prevención de Riesgos Laborales, Complejo Asistencial Universitario de León, León, Spain \\ ' Servicio de Prevención de Riesgos Laborales, Hospital Universitario Ramón y Cajal, Madrid, Spain

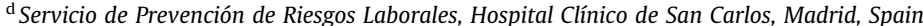 \\ e Servicio de Prevención de Riesgos Laborales, Complejo Asistencial Universitario de Palencia, Palencia, Spain \\ ${ }^{\mathrm{f}}$ Servicio de Prevención de Riesgos Laborales, Complejo Asistencial de Zamora, Zamora, Spain \\ ${ }^{g}$ Servicio de Prevención de Riesgos Laborales, Hospital Universitario Puerta de Hierro Majadahonda, Madrid, Spain \\ ${ }^{\mathrm{h}}$ Servicio de Medicina del Trabajo y Prevención de Riesgos Laborales, Hospital Universitario 12 de Octubre, Madrid, Spain \\ ${ }^{i}$ Servicio de Salud Laboral y Prevención. Hospital Universitario Fundación Jiménez Díaz Quirónsalud, Madrid, Spain \\ ${ }^{j}$ Servicio de Medicina del Trabajo, Hospital Universitario Gregorio Marañón, Madrid, Spain \\ ${ }^{\mathrm{k}}$ Servicio de Prevención de Riesgos Laborales, Hospital Clínico Universitario de Valladolid, Valladolid, Spain \\ ${ }^{1}$ Departamento de Estadística, Universidad de Salamanca, Salamanca, Spain \\ ${ }^{\mathrm{m}}$ UICEC, Complejo Asistencial Universitario de Salamanca - Instituto de Investigación Biomédica de Salamanca (IBSAL), Plataforma SCReN, Salamanca, Spain
}

\section{A R T I C L E I N F O}

\section{Article history:}

Received 6 August 2020

Received in revised form 16 November 2020

Accepted 1 December 2020

Available online 15 December 2020

\section{Keywords:}

Viable vaccine alternative

Conventional vaccine

Health centres dependent

Fendrix ${ }^{\circledR}$

Preventive clinical trial

\begin{abstract}
A B S T R A C T
Summary: Trial Design: An interventional, phase 4, single group assignment, without masking (open label), preventive clinical trial was carried out in health workers with biological risk in their tasks, who have been filed as non-responders to conventional vaccination against Hepatitis B.

Methods: 67 health workers with biological risk in their tasks, who have been filed as non-responders to conventional vaccination against Hepatitis B, were enrolled in the Clinical Trial. All participants were from 18 years up to 64 years old. Inclusion Criteria: NHS workers -including university students doing their internships in health centres dependent on the National Health System (inclusion of students is regulated and limited by specific instructions on labour prevention in each autonomous community)- classified as non-responders. The criteria defining them as non-responders to the conventional hepatitis $\mathrm{B}$ vaccine is anti HBsAb titers $<10 \mathrm{mUI} / \mathrm{ml}$ following the application of six doses of conventional vaccine at $20 \mu \mathrm{g}$ doses (two complete guidelines). The objective of this study was to provide Health workersstaff with an additional protection tool against hepatitis B infection, and to evaluate the efficacy of the adjuvanted vaccine in healthy non-responders to conventional hepatitis B vaccine. The primary outcome was the measurement of antibody antiHBs before the first Fendrix ${ }^{\circledR}$ dose and a month after the administration of each dose. Other outcome was collection of adverse effects during administration and all those that could be related to the vaccine and that occur within 30 days after each dose. In this study, only one group was assigned. There was no randomization or masking.

Results: The participants were recruited between April 13, 2018 and October 31, 2019. 67 participants were enrolled in the Clinical Trial and included the analyses. The primary immunisation consists of 4 separate $0.5 \mathrm{ml}$ doses of Fendrix ${ }^{\circledR}$, administered at the following schedule: 1 month, 2 months and 6 months from the date of the first dose. Once the positivity was reached in any of the doses, the participant finished the study and was not given the following doses. 68.66\% (46 out 67) had a positive response to first
\end{abstract}

\footnotetext{
* Corresponding authors.

E-mail addresses: jlbravo@saludcastillayleon.es (J.L. Bravo-Grande), ricardo.
} lopez@scren.es (R. López-Pérez). 
dose of Fendrix ${ }^{\circledR} .57 .14 \%$ (12 out 21 ) had a positive response to second dose of Fendrix ${ }^{\circledR}$. $22.22 \%$ (2 out 9 ) had a positive response to third dose of Fendrix and $42.96 \%$ ( 3 out 7 ) had a positive response to last dose of Fendrix ${ }^{\circledR}$. Overall, $94.02 \%$ (64 out 67 ) of participants had a positive response to Fendrix ${ }^{\circledR}$. No serious adverse event occurred.

Conclusions: The use of Fendrix ${ }^{\circledR}$, is a viable vaccine alternative for NHS workers classified as "nonresponders". Revaccination of healthy non-responders with Fendrix ${ }^{\circledR}$, resulted in very high proportions of responders without adverse events.

Trial registration: The trial was registered in the Spanish National Trial Register (REEC), ClinicalTrials.gov and inclusion has been stopped (identifier NCT03410953; EudraCT-number 2016004991-23).

Funding: GRS 1360/A/16: Call for aid for the financing of research projects in biomedicine, health management and socio-health care to be developed in the centres of the Regional Health Management of Autonomous Community of Castile-Leon. In addition, this work has been supported by the Spanish Platform for Clinical Research and Clinical Trials, SCReN (Spanish Clinical Research Network), funded by the Subdirectorate General for Research Evaluation and Promotion of the Carlos III Health Institute (ISCIII), through the project PT13/0002/0039 and project PT17/0017/0023 integrated in the State Plan for R\&D\&I 2013-2016 and co-financed by and the European Regional Development Fund (ERDF).

(C) 2020 Elsevier Ltd. All rights reserved.

\section{Introduction}

\subsection{Scientific background and rationale}

Independently of the current pandemic, working in health care entails exposure to numerous inherently associated risks even under normal circumstances. These include several biological risks which may vary widely according to the cases in question. One example is hepatitis $B$, which continues to cause many deaths worldwide.

Hepatitis B is the most serious type of viral hepatitis. It was first identified in 1963 by Blumberg, a US scientist. While analysing blood samples from populations around the world, he discovered the hepatitis B surface antigen in a sample from an Australian aborigine, hence he initially called it the Australian antigen. This laid the foundation for the study of a recently identified disease that had been known since ancient times as epidemic or catarrhal icterus [1-6]. Almost 20 years then passed until in 1981, the same Blumberg succeeded in obtaining a vaccine that was $95 \%$ effective against hepatitis B [7], followed by almost another decade of intensive vaccination campaigns until in 1991, the incidence of hepatitis B had fallen by approximately $82 \%$ in the United States [7-10].

Universal vaccination against this microorganism in children has improved in most developed countries. The vaccine has proved effective in reducing not only cases but also the long-term consequences of hepatitis $B$, such as chronic hepatitis, cirrhosis and hepatocellular carcinoma [2,6,9-12].

It is estimated that there are 250 million hepatitis B carriers in the world and that the virus kills about 900,000 people every year. According to the latest data from the European Union, the 25-44 age group presents the highest rate of new infections, followed by those aged $15-24$. Prevalence in Europe ranges between $0.1 \%$ in Ireland and $4.4 \%$ in Romania. Spain is one of the countries with a low endemicity of hepatitis $B$, presenting a cumulative prevalence of $\mathrm{HB}$ surface antigen (HBsAg) carriers of $0.8 \%$ [13]. In 2016, there were 530 cases of hepatitis B in Spain and the resulting incidence was 1.14 cases per 100,000 inhabitants. Due to its chronic nature, hepatitis B represents a major public health problem, not only because of its associated morbidity and mortality but also and fundamentally because $\mathrm{HBsAg}$ carriers constitute the main reservoir of the virus.

Although the vaccine is effective in $95 \%$ of individuals, no studies have been conducted to determine whether it remains effective after 10 years or if a booster is required. An American research team monitored 1578 individuals in Alaska (United States) who received three doses of the hepatitis B vaccine between 1981 and 1982 [14]. Fifteen years after vaccination, the team tested all subjects for antibodies that would protect them from infection and found that the number of antibodies against the virus had fallen since vaccination. The factors associated with a greater likelihood of continuing to present high antibody levels at 15 years were: being male, being older at the time of vaccination and having developed a high number of antibodies at the start [8-10,14-24].

In this regard, the study by Bruce et al. [17] provides widespread evidence that humoral immunity persists for $\geq 30$ years, however, it requires studies to determine the state of cellmediated immunity would be complementary and important, especially in those who lose anti-HBs and do not respond to a booster dose.

Although any individual without immunity to the hepatitis B virus (HBV) is susceptible to the disease, some groups are at greater risk due to high-risk practices, illness or simply personal contact. Health care staff comprise one of these groups, with a 210 times higher risk of infection than the general population. Transmission generally occurs through accidental exposure to patients' blood, primarily as a result of parenteral procedures $[3,11,18,20,25]$.

Since $5 \%$ of the general population does not respond to the conventional vaccine, and because antibody levels decrease over time, seroprotection against the virus remains uncertain. Therefore, it is necessary to find an alternative to protect health service staff who do not have antibodies, either because they never generated a response or because they no longer respond to the conventional vaccine. Fendrix ${ }^{\circledR}$, the hepatitis $B$ vaccine formulated with the new AS04 adjuvant (MPL + aluminum salts) by GlaxoSmithKline, may provide a good alternative [19,26-28]. The vaccine is indicated for patients with renal insufficiency, especially those on hemodialysis and pre-hemodialysis aged over 15 years old $[19,22,28-35]$. It has been reported that revaccination with Fendrix ${ }^{\circledR}$ has a high success rate in HIV patients who do not respond to other vaccination strategies against hepatitis B [30,34-35].

Compared with the conventional vaccine, the additional costs of administering Fendrix ${ }^{\circledR}$ to health service staff as a prophylactic against hepatitis $B$ are more than justified if we consider the mean costs entailed in monitoring and treating hepatitis B in health service staff infected through accidental exposure [36,37], combined with the associated sick leave [38-39]. In addition, in the case of accidental infection, the cost of a reported occupational accident would be lower in immunised than in non-immunised subjects [40]. 


\subsection{Specific objectives and hypothesis}

- Objectives

o To endow Spanish Health Service staff with additional protection against hepatitis B infection.

o To assess the efficacy of the adjuvanted vaccine in healthy subjects who do not respond to the conventional hepatitis $B$ vaccine.

- Hypothesis

o Administration of the Fendrix ${ }^{\circledR}$ vaccine is a viable alternative for Spanish Health Service staff classified as "nonresponders".

\section{Methods}

\subsection{Clinical study design}

\subsubsection{Description of the clinical study design}

We conducted a non-randomised, multi-centre, phase 4 clinical trial with a single group, without masking (open-label), administering medication for off-label use in Spanish Health Service staff whose work exposed them to biological risk and who had been classified as non-responders to the conventional hepatitis $\mathrm{B}$ vaccine.

\subsubsection{Significant changes in the methods after initiating the trial}

The main changes in this clinical trial concerned subject recruitment. As the initial rate of recruitment was lower than expected, we prolonged the recruitment time. This change modified overall study length and the dates of the trail stages. For similar reasons, we expanded our study population from the Autonomous Community of Castile-Leon Health Service (Spanish initials: SACYL) to the Spanish Health Service (Spanish initials: SNS) to enable inclusion of centres outside the Autonomous Community of Castile-Leon (Spanish initials: $\mathrm{CyL}$ ). This led to the inclusion of 6 centres from the autonomous community of Madrid. Lastly, to further facilitate recruitment, the inclusion criteria were modified to include health sciences students undertaking a work placement with the SNS. This inclusion of students was subject to and limited by the specific regulations on health and safety in each autonomous region.

This clinical study was approved by the medical research ethics committee at the University Hospital of Salamanca on 25 September 2017, for implementation at the participating centres (ref. 17/1311), and was authorised by the Spanish Agency for Medicines and Health Products (Spanish initials: AEMPS) on 16 October 2017 (EudraCT number: 2016-004991-23) for implementation in these hospitals. (For protocol see https://clinicaltrials.gov/ct2/show/ NCT03410953).

\subsection{Participants}

\subsubsection{Participant selection criteria}

In order to be recruited for this clinical trial, subjects had to work in the SNS -including university students undertaking work placements in SNS health centres (subject to and limited by the specific regulations on health and safety in each autonomous region) - and be of working age. In addition, they had to be free of any condition that contraindicated vaccination with Fendrix ${ }^{\circledR}$. They also had to meet the criteria for classification as a nonresponder to the conventional hepatitis $B$ vaccine: anti-HBs antibody titers of $<10 \mathrm{mIU} / \mathrm{ml}$ following administration of six $20 \mu \mathrm{g}$ doses of conventional vaccine (two complete series). All subjects signed an informed consent form.

The exclusion criteria were as follows: known allergy to the active ingredient or to any other of the drug components (included in section 6 of the summary of product characteristics); a past allergic reaction to any hepatitis B vaccine; or presenting a serious infection with fever at the time of recruitment. Subjects who did not give their informed consent were also excluded.

\subsubsection{Site (centres and institutions) where data were collected}

Eleven SNS hospitals participated in this clinical trial, and these are listed below:

- Complejo Asistencial Universitario de Salamanca. (Coordinating Center)

- Hospital Clínico Universitario de Valladolid

- Complejo Asistencial de Zamora

- Complejo Asistencial Universitario de León

- Complejo Asistencial Universitario de Palencia

- Instituto de Investigación Sanitaria Fundación Jiménez Díaz, Madrid.

- Hospital Clínico San Carlos, Madrid.

- Hospital Universitario 12 de Octubre, Madrid.

- Hospital Universitario Gregorio Marañón, Madrid.

- Hospital Universitario Puerta de Hierro, Madrid.

- Hospital Universitario Ramón y Cajal, Madrid.

\subsection{Interventions}

Fendrix ${ }^{\circledR}$ is a hepatitis $B$ vaccine that contains the HBsAg adjuvant (3-O-desacyl-4'-monophosphoryl lipid A and aluminum phosphate). This adjuvant system is known as AS04 and it boosts vaccine immunogenicity. An anti-HB antibody titre of $\geq 10 \mathrm{mIU} /$ $\mathrm{ml}$ correlates with protection against HBV infection.

Immunization consisted of a maximum of four $0.5 \mathrm{ml}$ doses of Fendrix ${ }^{\circledR}$ administered as follows: 1, 2 and 6 months after the date of the first dose, by intramuscular injection of a $20 \mu \mathrm{g}$ dose in the deltoid muscle, as indicated in the Fendrix ${ }^{\circledR}$ summary of product characteristics. We conducted a serological analysis about 30 days after administration of each dose. If subjects presented seroprotection (anti-HB $\geq 10 \mathrm{mIU} / \mathrm{ml}$ ), administration of subsequent doses was suspended.

The Fendrix ${ }^{\circledR}$ vaccine lots used in this trial were: AFENA027BC (01/2020), AFENA028AI (05/2020) and AFENA029AC (09/2020).

We observed precautionary measures as regards the product contraindications and warnings indicated by the manufacturer and AEMPS recommendations for off-label use.

Thirty days after administration of each vaccine dose, we took a venous blood sample to determine anti-HBs titres. Serum samples were analysed immediately in the certified reference laboratory at each participating hospital, extracting the sample according to each laboratory's technical instructions. Anti-HBs values above $1000 \mathrm{mIU} / \mathrm{ml}$ were assigned a value of $1000 \mathrm{mIU} / \mathrm{ml}$. The lower limit of detection for the trial was $3 \mathrm{mIU} / \mathrm{ml}$. All values below the lower limit of detection were assigned a value of $3 \mathrm{mIU} / \mathrm{ml}$.

Adverse effects were recorded during vaccine administration, noting all those that could be related to the vaccine and had appeared in the 30 days following each dose.

\subsubsection{Results}

After measuring the anti-HBs antibody titre before the first dose and one month after administration of each dose, the results for anti-HBs antibody titres were analysed to determine immune response to the adjuvanted vaccine. Anti-HBs titres greater than or equal to $10 \mathrm{mIU} / \mathrm{ml}$ were classified as positive.

To assess results for adjuvanted vaccine safety and clinical tolerance, adverse effects were recorded during administration, noting all those that could be related to the vaccine and had appeared within 30 days after each dose.

As secondary objectives, we analysed biochemical data collected immediately prior to administering the first vaccine to 
determine participant characteristics. We also assessed the number of doses necessary to achieve a positive response. In addition, we analysed the influence of sex and age on the results.

\subsubsection{Sample size}

The total number of staff at the 11 centres participating in this clinical trial was approximately 47,650 at the time of conducting the trial. In an analysis that included 2620 eligible adults who had participated in 11 studies conducted in 10 countries, Van Der Meeren [41] described a 94.5\% response rate to the conventional vaccine. Consequently, we estimated a target population for our trial of 2621 subjects. Raheja and colleagues [42] have indicated that for various reasons, about one-third of workers will refuse to participate in this type of trial, which would reduce our target population to 1730 .

A literature review indicated that the mean percentage of workers who do not respond to the Fendrix ${ }^{\circledR}$ vaccine ranges from below $1 \%$ to $10 \%[7,15,19,29,32,35]$. This would noticeably affect sample size calculation. Taking $5 \%$ as the mean percentage of workers who do not respond to Fendrix ${ }^{\circledR}$ and assuming an error margin of $\pm 1 \%$, we required 70 subjects in total, with a range of $57-83$, in order to achieve a $95 \%$ confidence level with a maximum acceptable error margin of $5 \%$.

\subsubsection{Randomization and masking}

This was an open clinical trial without control group or masking.

\subsubsection{Statistical analysis}

We used two statistical methods to compare groups:

When both the variable of interest and the secondary variables were qualitative, we entered them into contingency tables and used Fisher's exact test to determine any dependency between both variables.

When the variable of interest was numeric and the secondary variables were qualitative, we used the Wilcoxon rank-sum test, since numeric variables do not generally come from normally distributed populations and therefore the classic parametric tests arenot recommended. We used the Shapiro-Wilks test to determine normality, due to its higher power compared to other similar tests [43].

In all analyses, we eliminated missing values.

To describe the qualitative variables, we used frequency tables and bar graphs, obtaining confidence intervals for the first category of each variable.

Means and standard deviations were used to describe numeric variables, but also quartiles, due to the absence of normality, both globally and by category for some qualitative variables. We also estimated confidence intervals for the population mean of each variable, assuming unknown variance.

In addition, we calculated Spearman correlations for numeric variables to determine dependency between them. Once again, a non-parametric method was used due to the absence of normality.

\section{Results}

\subsection{Participant flow chart}

\subsubsection{Recruitment}

3.1.1.1. Dates that define the periods of recruitment and followup. From 26 April 2018, the date of the inclusion of the first subject, to 11 June 2019, the date of the inclusion of the last subject, 94 individuals were assessed for eligibility, of whom 67 were included in the present study (Fig. 1). Follow-up continued until 31 October 2019.
On 14 November 2019, the trial was concluded with 67 recruited subjects. Of the 94 subjects assessed for eligibility, 27 did not agree to participate in the trial and declined to sign an informed consent form.

In the absence of losses or exclusions, data analysis was conducted with 67 subjects.

\subsubsection{Baseline data}

3.1.2.1. Baseline demographic and clinical characteristics. The possible factors that could alter our primary result (e.g. age, baseline anti-HBs titre, diabetes, body mass index) were in line with those of a normal healthy population of non-responders to the conventional vaccine (Table 1, Table Anx1 and Table Anx2), with the exception of sex, whereby the population presented a marked shift towards women, reflecting the current composition of the health worker population in the Spanish Health Service.

\subsubsection{Numbers analysed}

As described in the interventions section, the vaccine was only administered to subjects whose previous serology was negative. Thus, the first dose was administered to 67 subjects, the second dose to 21 subjects, the third to 9 subjects and the fourth to 7 subjects.

3.1.3.1. Distribution of numeric variables. We used the ShapiroWilks test [43] to determine whether any of the numeric variables comes from a normal distribution (Gaussian). The hypotheses were:

- $\mathrm{H}_{0}$ : the sample variable comes from a normally distributed population

- $\mathrm{H}_{1}$ : the sample variable does not come from a normally distributed population

Most of the numeric variables (except "height" and "creatinine") did not present a normal distribution, with a significance of $\leq 5 \mathrm{e}-02(0.05)$ (see Table 2).

\subsubsection{Results and estimation}

Of the 67 subjects participating in this trial, 63 (94.03\%) attained anti-HBs levels of $\geq 10 \mathrm{mIU} / \mathrm{ml}$, indicating seroprotection against HBV (Table 3).

A cumulative analysis of the response data indicated a gradual increase in subjects reaching seroprotection: $68.66 \%$ with the first dose, $86.57 \%$ with the second dose, $89.55 \%$ with the third dose and 94.03\% with the fourth dose (Fig. 2).

We did not detect any serious adverse effects during this clinical trial. Most of the adverse effects observed occurred after administering the first dose. Some 30\% of vaccinations (32 out of 104) gave rise to adverse effects, and of these, $78.12 \%$ were related to the medication under study (Table 4). The symptoms were transient and almost all resolved spontaneously within 1 week.

By symptom, the most common effect $(59.38 \%)$ was pain at the injection site. Seven adverse effects were not associated with the medication, and the most common of these was "malaise". Table 4 gives all the data obtained on adverse effects.

\subsubsection{Secondary analysis}

We found a relationship between sex and response to the first vaccine dose. This was determined by performing the Wilcoxon rank-sum test with continuity correction, where the hypotheses to test were $\mathrm{H}_{0}$ : Anti-HBs (men) $\geq$ Anti-HBs (women) and $\mathrm{H}_{1}$ : Anti-HBs (men) < Anti-HBs (women). The test yielded a p-value of 0.037 ( $W=318.5$ ), which is lower than 0.05 , thus $\mathrm{H}_{0}$ is rejected and $\mathrm{H}_{1}$ accepted, indicating that men presented a lower level of Anti-HBs (Fig. 3). 


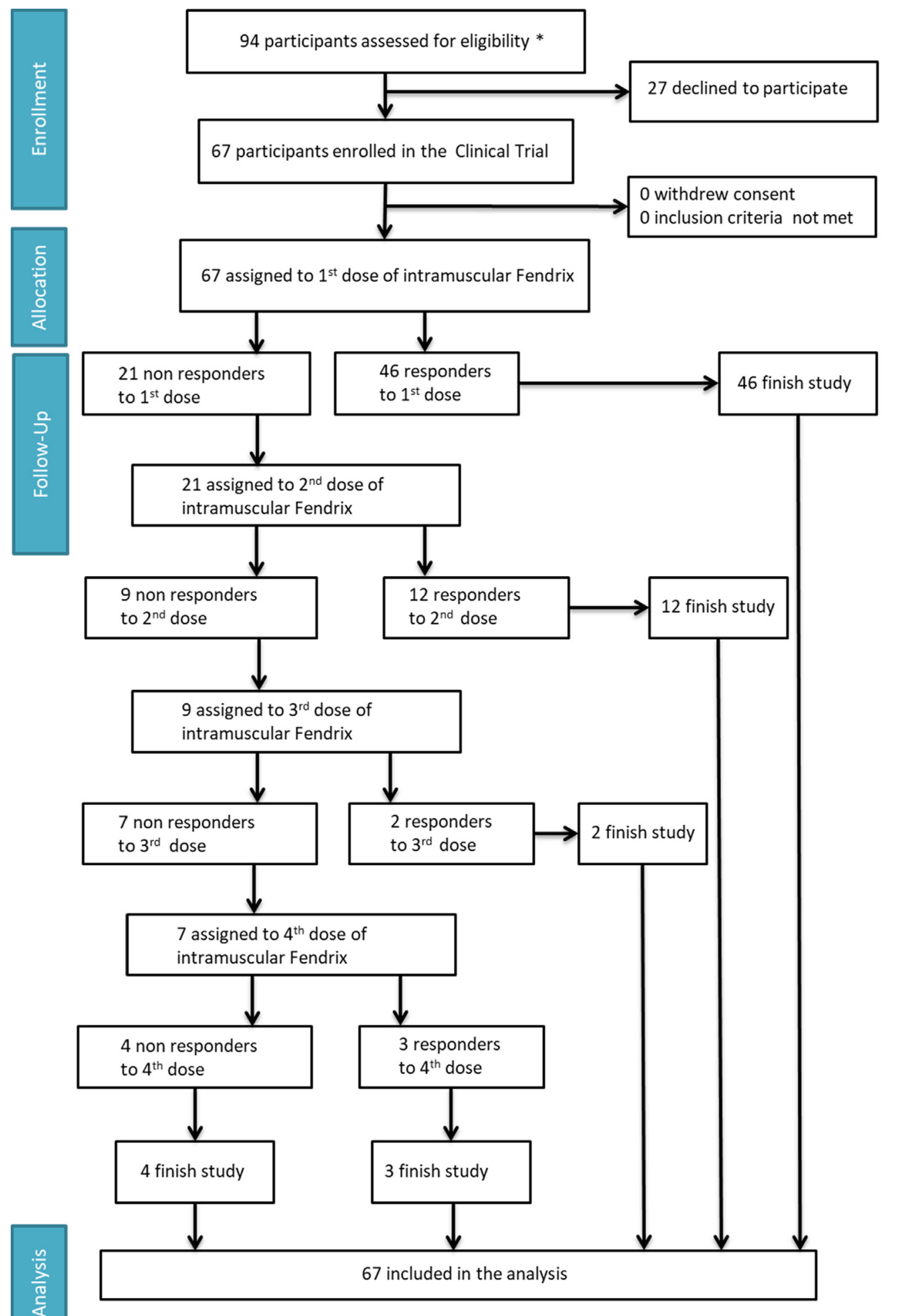

*Based on estimates from centres who provided the participants.

Fig. 1. Flow chart.

This effect was not observed for the following doses administered, probably because of the low " $n$ " when these doses were administered. This effect has been described previously for other hepatitis B vaccines. $[20,44]$

Table 5 shows the quantitativevariables that present significant differences by the sex categories. In all the variables, except
"AntihHbS visit 2", the values in male are significantly higher than in female.

3.1.5.1. Spearman correlation matrix. We found the following significant correlations ( $\mathrm{p}$-value $\leq 0.05$ ) as shown in Fig. Anx1:

- Age and Glucose, Urea, GGT, Visit_2_AntiHbS. 
Table 1

Demographic and clinical baseline characteristics.

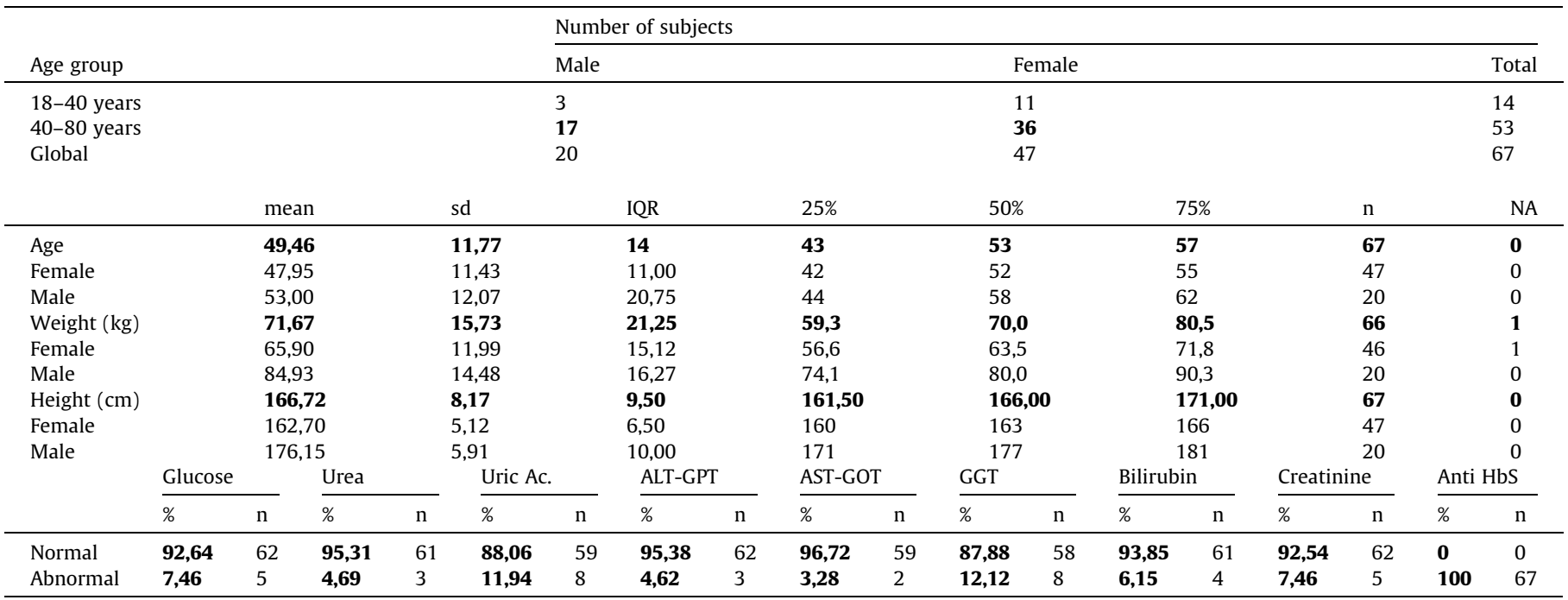

Table 2

Distribution of the numerical variables analyzed.

\begin{tabular}{ll}
\hline & Shapiro_Wilks_p.value \\
\hline Age & $7.851978 \mathrm{e}-05$ \\
Weight $(\mathrm{Kg})$ & $1.375092 \mathrm{e}-03$ \\
Height $(\mathrm{cm})$ & $6.245423 \mathrm{e}-02$ \\
Selection Visit AntiHbS & $2.611177 \mathrm{e}-15$ \\
Selection Visit Glucose & $1.172472 \mathrm{e}-02$ \\
Selection Visit Uric Acid & $3.507400 \mathrm{e}-02$ \\
Selection Visit Urea & $1.281404 \mathrm{e}-02$ \\
Selection Visit Creatinine & $1.598279 \mathrm{e}-01$ \\
Selection Visit Bilirubin & $8.114063 \mathrm{e}-10$ \\
Selection Visit AST_GOT & $4.851082 \mathrm{e}-11$ \\
Selection Visit ALT_GPT & $1.844211 \mathrm{e}-11$ \\
Selection Visit GGT & $4.085315 \mathrm{e}-13$ \\
Visit 2_AntiHbS & $2.638358 \mathrm{e}-11$ \\
Visit 3_AntiHbS & $6.580777 \mathrm{e}-06$ \\
Visit 4_Serolog_AntiHbS & $7.054380 \mathrm{e}-05$ \\
Final visit AntiHbS & $1.000965 \mathrm{e}-05$
\end{tabular}

Consequently, we used non-parametric tests with these variables to test other associated hypotheses.

- Weight and Height, Selec_visit_AntiHbS, Glucose, Uric acid, Creatinine, ALT_GPT, GGT.

- Height and Uric acid, Creatinine, Bilirubin.

- Glucose and Uric acid, GGT.

- Uric Acid and ALT_GPT, GGT.

- Urea and Creatinine.

- Creatinine and GGT

- AST_GOT and ALT_GPT.

- ALT_GPT and GGT, Visit_2_AntiHbS.

- GGT and Visit_2_AntiHbS.

- Visit_3_AntiHbS and Visit_4_AntiHbS.
We also analysed the influence of age on vaccine response and observed a significant correlation $(r=-0.37$ with $p$-value $=0.0019)$ between age and response to the first dose. This finding indicates an inverse correlation between variables, whereby the higher the age, the lower the response (Fig. 4). This effect was not observed for the following doses administered, probably because of the low " $n$ " when these doses were administered.

3.1.5.2. Contingency tables and test of independence. To study dependence between two qualitative variables, we performed Fisher's exact test, where the hypotheses were:

o $\mathrm{H}_{0}$ : Variables are independent

o $\mathrm{H}_{1}$ : Variables are dependent

The most relevant pair of variables that yielded a significant pvalue was:

- "Anti-HBs visit 2" and "Adverse effects visit 2", pvalue $=0.0005149$.

This indicates that the adverse effects reported following administration of the first dose appear to be linked to not attaining seroprotection (Fig. 5). This effect was not observed for the following doses administered, probably because of the low " $n$ " when these doses were administered.

No relationship was found between the biochemical parameters analysed -glucose, uric acid, urea, creatinine, bilirubin, AST-GOT, ALT-GPT and GGT- and the response to vaccination with Fendrix ${ }^{\circledR}$.

3.1.5.3. Influence of the time between the last dose of conventional vaccine and revaccination with Fendrix on the response to vaccination. We analyzed the influence of the time elapsed between pre-

Table 3

Vaccination response by dose.

\begin{tabular}{|c|c|c|c|c|}
\hline Dose & Vaccinated & Responders & Non-responders & $\%$ response \\
\hline First & 67 & 46 & 21 & $68,66 \%$ \\
\hline Second & 21 & 12 & 9 & $57,14 \%$ \\
\hline Third & 9 & 2 & 7 & $22,22 \%$ \\
\hline Fourth & 7 & 3 & 4 & $42,86 \%$ \\
\hline Total & 67 & 63 & 4 & $94,03 \%$ \\
\hline
\end{tabular}




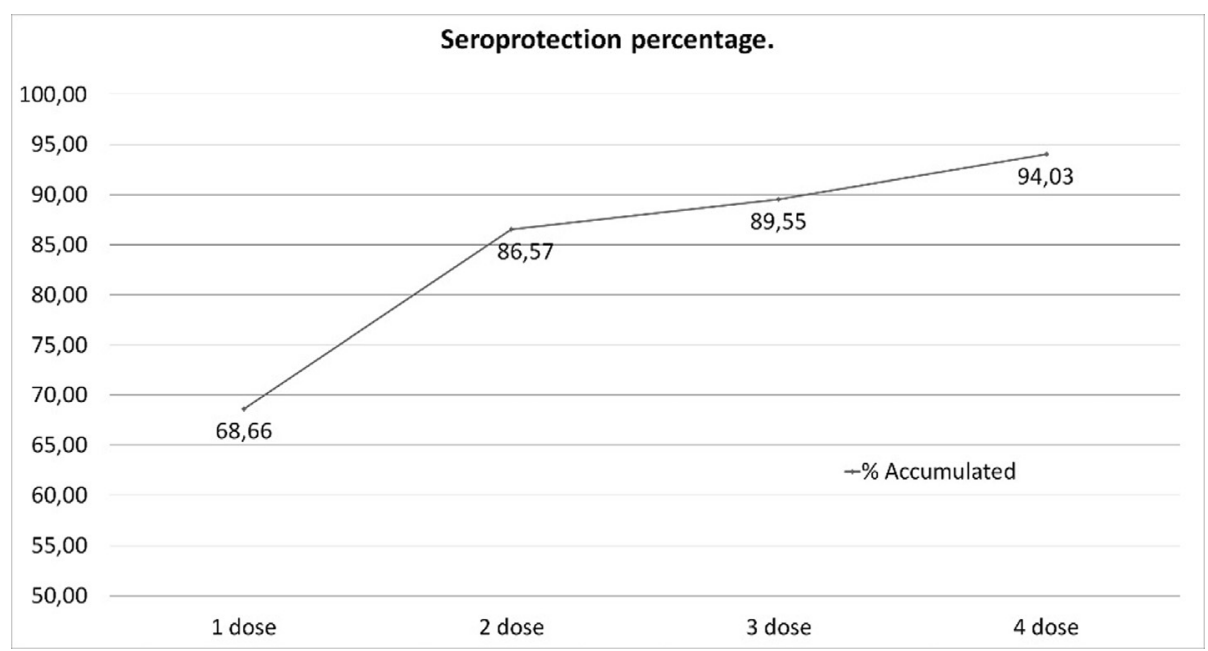

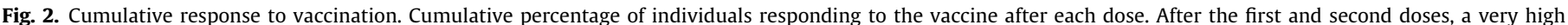
percentage of positive response to the vaccine is achieved.

Table 4

Adverse Events (AE) found during the trial.

\begin{tabular}{|c|c|c|c|c|c|c|c|c|c|c|}
\hline & \multicolumn{2}{|l|}{ Dose 1} & \multicolumn{2}{|c|}{ Dose 2} & \multicolumn{2}{|l|}{ Dose 3} & \multicolumn{2}{|l|}{ Dose 4} & \multirow[t]{2}{*}{ Total n } & \multirow[t]{2}{*}{ Overall \% } \\
\hline & $\%$ & $\mathrm{n}$ & $\%$ & $\mathrm{n}$ & $\%$ & $\mathrm{n}$ & $\%$ & $\mathrm{n}$ & & \\
\hline Subjects with AE & 31,30 & 21 & 28,57 & 6 & 11,11 & 1 & 14,28 & 1 & & \\
\hline Detected AE & & 24 & & 6 & & 1 & & 1 & 32 & \\
\hline \multicolumn{11}{|l|}{ Related to Medication } \\
\hline Yes & 76,20 & 19 & 83,33 & 5 & 100,00 & 1 & 0,00 & 0 & 25 & 78.12 \\
\hline No & 23,80 & 5 & 13,66 & 1 & 0,00 & 0 & 100,00 & 1 & 7 & 21.87 \\
\hline \multicolumn{11}{|l|}{ Type } \\
\hline Puncture Zone Pain & 58,33 & 14 & 66,67 & 4 & 100,00 & 1 & 0,00 & 0 & 19 & 59,38 \\
\hline Discomfort & 12,50 & 3 & 16,67 & 1 & 0,00 & 0 & 0,00 & 0 & 4 & 12,50 \\
\hline Fatigue & 12,50 & 3 & 0,00 & 0 & 0,00 & 0 & 0,00 & 0 & 3 & 9,38 \\
\hline Diarrhea & 8,33 & 2 & 0,00 & 0 & 0,00 & 0 & 0,00 & 0 & 2 & 6,25 \\
\hline Paresthesia in the puncture area & 8,33 & 2 & 0,00 & 0 & 0,00 & 0 & 0,00 & 0 & 2 & 6,25 \\
\hline Cramp & 0,00 & 0 & 16,67 & 1 & 0,00 & 0 & 0,00 & 0 & 1 & 3,13 \\
\hline Diverticulitis & 0,00 & 0 & 0,00 & 0 & 0,00 & 0 & 100,00 & 1 & 1 & 3,13 \\
\hline
\end{tabular}

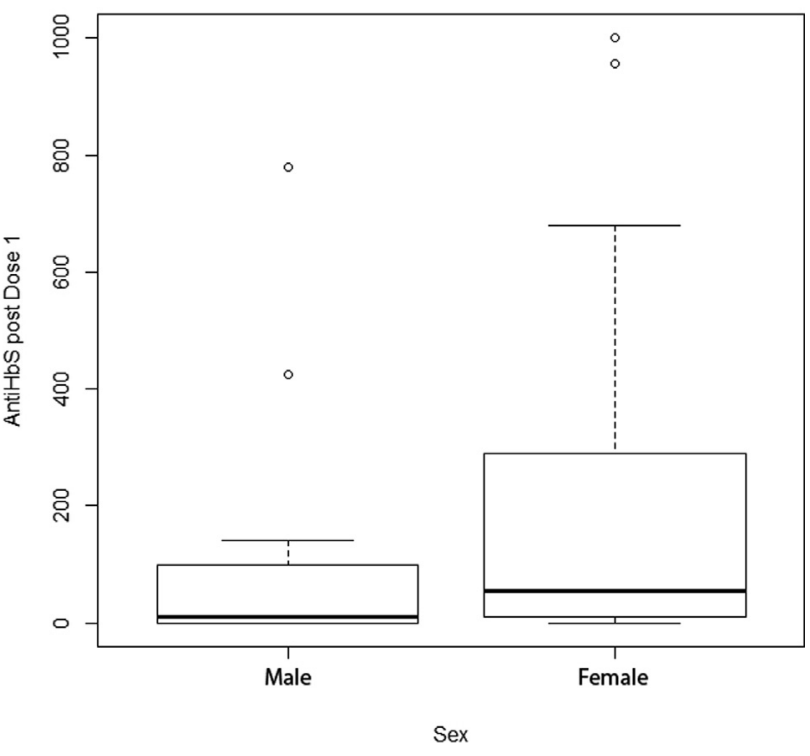

Fig. 3. Response to the first dose of vaccine; comparative by sex. Boxplot of the distributions by sex of 'AntiHbS after dose 1' where we observe that males present lower values and with less dispersion than females.
Table 5

Wilcoxon test of the quantitative variables that present significant differences by sex.

\begin{tabular}{lll}
\hline & Sex & \\
\cline { 2 - 3 } & W & p-value \\
\hline Age & 627 & $3,10 \mathrm{E}-02$ \\
Weight $(\mathrm{kg})$ & 789,5 & $4,39 \mathrm{E}-06$ \\
Height $(\mathrm{Cm})$ & 909,5 & $1,71 \mathrm{E}-09$ \\
Uric acid (mg/dL) & 751 & $1,20 \mathrm{E}-04$ \\
Urea $(\mathrm{mg} / \mathrm{dL})$ & 521 & $2,40 \mathrm{E}-02$ \\
Creatinine (mg/dL) & 874 & $3,19 \mathrm{E}-08$ \\
Bilirubin $(\mathrm{mg} / \mathrm{dL})$ & 620,5 & $1,50 \mathrm{E}-02$ \\
AST-GOT (U/L) & 566 & $1,60 \mathrm{E}-02$ \\
ALT-GPT (U/L) & 649 & $4,70 \mathrm{E}-03$ \\
GGT (U/L) & 690,5 & $1,30 \mathrm{E}-03$ \\
AntiHbS Visit 2 (mUI/ml) & 318,5 & $3,70 \mathrm{E}-02$ \\
\hline
\end{tabular}

study vaccination with the conventional vaccine and vaccination with Fendrix for the trial. We do not see any relationship between the time between vaccines and the response to vaccination with Fendrix. Analyzing the final response to Fendrix, we find two very unbalanced groups, to which after applying "Wilcoxon rank sum test with continuity correction" we obtain W $=144$ and p-value 


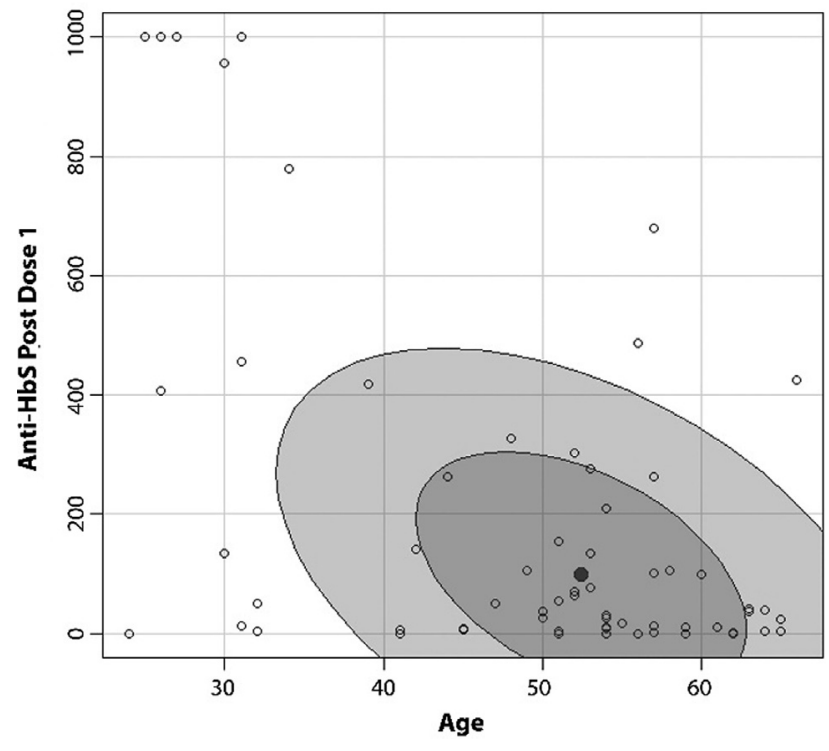

Fig. 4. Response to the first dose of vaccine; Influence of age. Scatterplot between 'age' and 'AntiHbS after dose 1' showing that low levels of AntiHbS are mainly concentrated in individuals aged between 50 and 60 years.

$=0.6003$ showing no significant differences between both groups (Fig. Anx2).

Analyzing the initial response to Fendrix, we find two more balanced groups, to which after applying "Wilcoxon rank sum exact test" we obtain $\mathrm{W}=540$ and p-value $=0.3592$, not showing significant differences between the two groups (Fig. Anx3).

3.1.5.4. Dependence between response and other factors. Finally, we analyzed whether any immunosuppressive conditions, such as the use of steroids or immunosuppressive diseases, or whether previous or current pathologies, or if the concomitant medication in the study had any influence on the response to revaccination with Fendrix. These data were analyzed with the total response to vaccination and the response to the first dose of Fendrix.
The only relevant data resulting from the analysis carried out is that there is a dependency between "Global response" and "Possible conditioning pathology -YES/NO-" ( $\mathrm{p}$-value $=0.0063$ ) (Fig. Anx4) and between 'Global response' and "Concomitant medication -YES/NO- "(p-value = 0.0092) (Fig. Anx5).

There are insufficient data to determine whether these associations are due to a specific pathology or to a specific drug.

\subsubsection{Adverse effects (risk)}

The 67 participants reported 32 adverse effects (AEs), of which 25 were adverse reaction (AR). None of the adverse effects reported were considered serious and all patients recovered from their corresponding adverse effect, suggesting a low risk of serious adverse effects and an acceptable risk in relation to non-serious adverse effects, which corresponded to those already identified in the Technical Data Sheet.

Four subjects had diabetes mellitus type 2 prior to participating in the trial. Van Der Meeren et al. [45] reported that the reactogenicity and safety profile of the hepatitis $B$ vaccine (Engerix$\mathrm{B}^{\mathrm{TM}}$ ) appeared similar in controls and patients with hepatitis $\mathrm{B}$ and was consistent with the experience of the vaccine). Two of them did not reach a response after 4 doses of Fendrix ${ }^{\circledR}$ and the others attained seroprotection after the fourth dose. Another non-responder had autoimmune hepatitis prior to participating in the trial and the last non-responder did not present any pathologies of interest.

3.1.6.1. Risk-benefit assessment. An analysis of the results revealed no evidence of significant, identifiable or potential risks related to administration of Fendrix ${ }^{\circledR}$ in the study population (health service staff and university students whose work in the SNS exposed them to biological risk and who had been classified as nonresponders to the hepatitis $B$ vaccine), beyond those already indicated in the Technical Data Sheet.

\subsubsection{Discussion}

3.1.7.1. Limitations. Conducting the clinical trial in accordance with the instructions of the Spanish Agency for Medicines and Health Products (AEMPS) generated a slight difficulty. The AEMPS has ruled that Fendrix ${ }^{\circledR}$ should be administered as described in the summary of product characteristics. Our trial observed these spec-

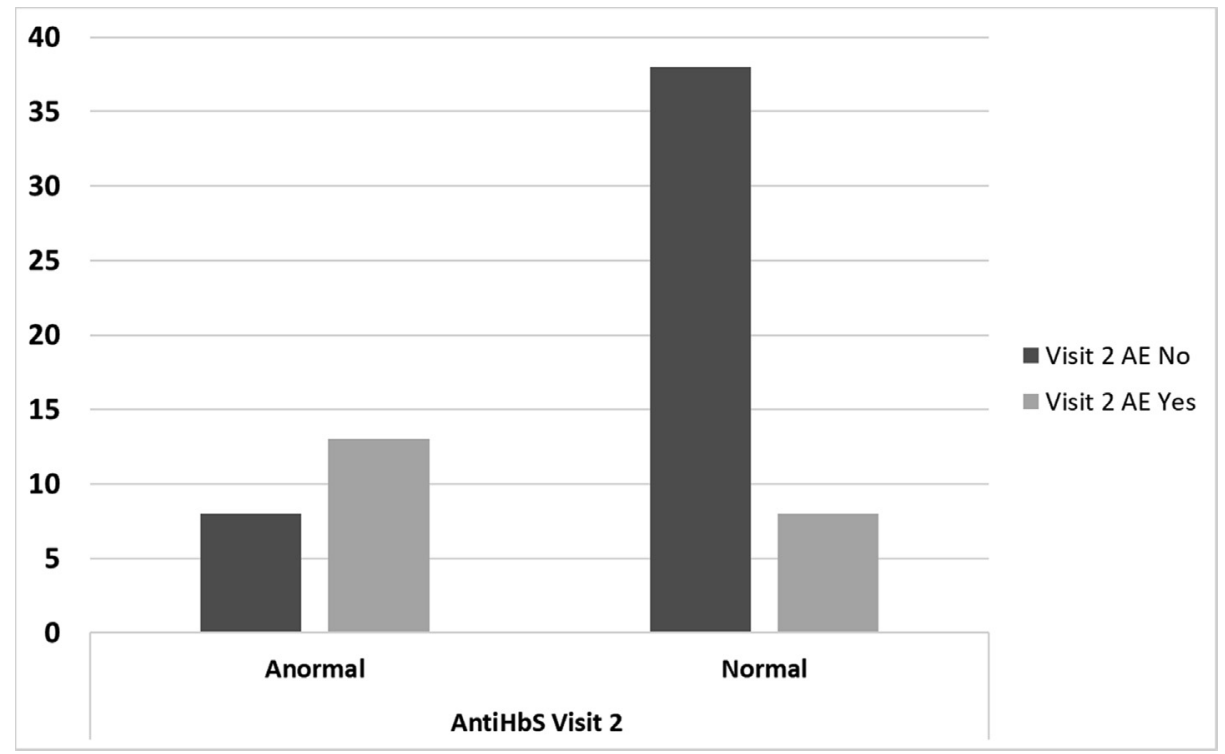

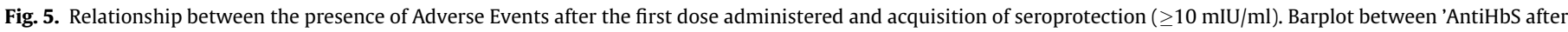
dose 1 ' and 'Presence of adverse events' showing that individuals with abnormal values of 'AntiHbs after dose 1 ' had adverse events. 
ifications, which required an effort on the part of all those involved. However, this limitation endowed our trial with added value.

One of the greatest difficulties we encountered while conducting this trial was recruitment, as samples from all hospitals were small. This resulted in the need to extend recruitment to additional centres in order to obtain an optimal study sample.

We also encountered a lack of information concerning staff who do not respond to vaccination. The exclusion of the Fendrix ${ }^{\circledR}$ vaccine for off-label use, or recent approval for use in patients with immunodeficiency, diverts attention away from non-responder staff. Consequently, treatment with specific immunoglobulin remains the only possible option to treat a worker potentially infected with the hepatitis B virus, despite the existence of a much better alternative: the Fendrix ${ }^{\circledR}$ vaccine.

Lastly, we also encountered some isolated difficulties as regards organizing all trial visits and their dates for various reasons, including vacations and sick leave. Although this posed an added difficulty, it was resolved through study coordination.

3.1.7.2. Extrapolation. The off-label use of Fendrix ${ }^{\circledR}$ described here will enable its administration to many potential beneficiaries if the AEMPS approves such off-label use. These include:

o Health service staff

o Non-health service staff

o Employees in workplaces related to health care

o State security forces

o Prison officers

Our results pave the way for replicating the trial in other countries.

3.1.7.3. Interpretation. From the perspective of health, Fendrix ${ }^{\circledR}$ represents a viable alternative vaccine for SNS staff classified as "non-responders".

As regards costs, according to previous publications, the "cost" of the vaccine is offset by savings in:

- The cost of follow-up after a biological accident.

- The cost of administering specific immunoglobulin.

- The personal cost to the worker concerned.

- The level of response to a single dose.

\section{Other information.}

Trial registration: The trial was registered in the Spanish National Trial Register (REEC), ClinicalTrials.gov and inclusion has been stopped (identifier NCT03410953; EudraCT-number 2016004991-23).

Protocol: For protocol see https://clinicaltrials.gov/ct2/show/ NCT03410953

Funding: GRS 1360/A/16. Call for aid for the financing of research projects in biomedicine, health management and sociohealth care to be developed in the centers of the Regional Health Management of Castilla y León. In addition, this work has been supported by the Spanish Platform for Clinical Research and Clinical Trials, SCReN (Spanish Clinical Research Network), funded by the Subdirectorate General for Research Evaluation and Promotion of the Carlos III Health Institute (ISCIII), through the project PT13/0002/0039 and project PT17/0017/0023 integrated in the State Plan for R\&D\&I 2013-2016 and co-financed by and the European Regional Development Fund (ERDF).

Ethics approval and consent to participate: All procedures in the study were performed in accordance with the ethical standards of the institutional research committee and with the 1964 Declaration of Helsinki and its later amendments or comparable ethical standards. Informed consent was obtained from all individual participants included in the study.

\section{CRediT authorship contribution statement}

José L. Bravo-Grande: Conceptualization, Methodology, Supervision, Funding acquisition, Writing - original draft. M. Asunción Blanco-González: Investigation, Resources. José M. de la TorreRobles: Investigation, Resources. Marita P. Asmat-Inostrosa: Investigation, Resources. Marina Fernández-Escribano: Investigation, Resources. Itria Mirabella Villalobos: Investigation, Resources. M. Covadonga Caso-Pita: Investigation, Resources. Marina Hervella-Ordoñez: Investigation, Resources. Luis M. Cañibano Cimas: Investigation, Resources. José M. de la FuenteMartín: Investigation, Resources. M. Luisa Rodríguez de la Pinta: Investigation, Resources. Jenry Ricardo Borda Olivas: Investigation, Resources. Carmen Muñóz-Ruipérez: Investigation, Resources. Miguel A. Alonso López: Investigation, Resources. Teresa del Campo-Balsa: Investigation, Resources. M. Antonieta Ramírez Pérez: Investigation, Resources. Ignacio SánchezArcilla: Investigation, Resources. Marco Marzola-Payares: Investigation, Resources. Fernando Rescalvo-Santiago: Investigation, Resources. Miguel de Paula-Ortiz: Investigation, Resources. José M. Sánchez-Santos: Formal analysis, Data curation, Visualization. Ricardo López-Pérez: Methodology, Validation, Data curation, Project administration, Visualization, Writing - original draft, Writing - review \& editing.

\section{Declaration of Competing Interest}

The authors declare that they have no known competing financial interests or personal relationships that could have appeared to influence the work reported in this paper.

\section{Acknowledgements}

We want to thank you for your participation in the Clinical Trial IBS-VACANTIB-1701 to:

Elena Alonso Vicente, Rosa M. Sánchez Cívicos y Gonzalo Aicardi Carrillo (Complejo Asistencial Universitario de Salamanca), María Casares del Rio, Luis Alonso herrero, Carmen Espadas Lazo, Marta Olmedo Ordás, Teresa Fernández García, Raquel García Fernández, Javier González Espinel y Victoria Barajas Lorenzo (Complejo Asistencial Universitario de León), María D. Moro González, Rebeca García Rojo, María E. Cabrera Sanz y Mariela Gonçalves De Freitas (Hospital Clínico Universitario de Valladolid), Ruth Fernández Aladrén, Lucía Alonso Andrés y María R. García Díez (Complejo Asistencial de Zamora), Eugenio Roa Seseña, Cristina Frechoso Guerrero, Juan J. Estebánez Marcos, Antonio Martin García, Mercedes Iribarren Torres y Rosario de Los Mozos Prieto (Complejo Asistencial Universitario de Palencia), Ana I. García Vaquero, Carmen Riesgo Gómez-Roso y Tito L. Lizárraga Hurtado (Hospital Universitario 12 de Octubre), Verónica Montes García, Beatriz Jiménez Martínez y María C. de la Iglesia Pérez (Hospital Universitario Gregario Marañón).

We also want to thank Patricia Rodríguez Fortúnez, Pharmacovigilance Manager at SCReN at Clinical Research and Clinical Trial Unit (UICEC) of Hospital Universitario de Canarias, for advice on the planning and implementation of pharmacovigilance plans.

\section{ORCID iDs}

Ricardo López Pérez: https://orcid.org/0000-0001-9495-8604. 


\section{Appendix A. Supplementary material}

Supplementary data to this article can be found online at https://doi.org/10.1016/j.vaccine.2020.12.006.

\section{References}

[1] Gerlich WH. Medical virology of hepatitis B: how it began and where we are now. Virol J. 2013; 10:239.

[2] Seeff LB, Beebe GW, Hoofnagle JH, Norman JE, Buskell-Bales Z, Waggoner JG, et al. A serologic follow-up of the 1942 epidemic of post-vaccination hepatitis in the United States Army. N Engl J Med 1987:316(16):965-70.

[3] Chen W, Gluud C. Vaccines for preventing hepatitis B in health-care workers. Cochrane Database Syst Rev 2005(4):CD000100.

[4] Tabor E, Buynak E, Smallwood LA, Snoy P, Hilleman M, Gerety RJ. Inactivation of hepatitis B virus by three methods: treatment with pepsin, urea, or formalin. J Med Virol 1983;11(1):1-9.

[5] Stephenne J. Recombinant versus plasma-derived hepatitis B vaccines: issues of safety, immunogenicity and cost-effectiveness. Vaccine 1988;6(4):299-303.

[6] Lemon SM, Thomas DL. Vaccines to prevent viral hepatitis. N Engl J Med 1997;336(3):196-204.

[7] Szmuness W, Stevens CE, Zang EA, Harley EJ, Kellner A. A controlled clinical trial of the efficacy of the hepatitis B vaccine (Heptavax B): a final report. Hepatology 1981;1(5):377-85.

[8] McMahon BJ, Helminiak C, Wainwright RB, Bulkow L, Trimble BA, Wainwright K. Frequency of adverse reactions to hepatitis B vaccine in 43,618 persons. Am J Med 1992;92(3):254-6.

[9] André FE. Overview of a 5-year clinical experience with a yeast-derived hepatitis B vaccine. Vaccine. 1990; 8 Suppl:S74-8; discussion S9-80.

[10] Shepard CW, Simard EP, Finelli L, Fiore AE, Bell BP. Hepatitis B virus infection: epidemiology and vaccination. Epidemiol Rev 2006;28:112-25.

[11] Averhoff F, Mahoney F, Coleman P, Schatz G, Hurwitz E, Margolis H. Immunogenicity of hepatitis B Vaccines. Implications for persons at occupational risk of hepatitis B virus infection. Am J Prev Med 1998;15(1):1-8.

[12] Centers for Disease C. Recommendation of the Immunization Practices Advisory Committee (ACIP). Inactivated hepatitis B virus vaccine. MMWR Morb Mortal Wkly Rep 1982; 31(24):317-22, 27-8.

[13] Kmet Lunacek N, Poljak M, Maticic M. Distribution of hepatitis B virus genotypes in Europe and clinical implications: a review. Acta Dermatovenerol Alp Pannonica Adriat 2018;27(3):141-6.

[14] McMahon BJ, Bruden DL, Petersen KM, Bulkow LR, Parkinson AJ, Nainan O, et al. Antibody levels and protection after hepatitis B vaccination: results of a 15-year follow-up. Ann Intern Med 2005;142(5):333-41.

[15] Fabrizi F, Cerutti R, Nardelli L, Tripodi F, Messa P. HBV vaccination with Fendrix is effective and safe in pre-dialysis CKD population. Clin Res Hepatol Gastroenterol 2019.

[16] Yang S, Tian G, Cui Y, Ding C, Deng M, Yu C, et al. Factors influencing immunologic response to hepatitis B vaccine in adults. Sci Rep 2016;6:27251.

[17] Bruce MG, Bruden D, Hurlburt D, Zanis C, Thompson G, Rea L, et al. Antibody levels and protection after hepatitis B vaccine: results of a 30-year follow-up study and response to a booster dose. J Infect Dis 2016;214(1):16-22.

[18] Leuridan E, Van Damme P. Hepatitis B and the need for a booster dose. Clin Infect Dis 2011;53(1):68-75.

[19] Tong NK, Beran J, Kee SA, Miguel JL, Sanchez C, Bayas JM, et al. Immunogenicity and safety of an adjuvanted hepatitis B vaccine in pre-hemodialysis and hemodialysis patients. Kidney Int 2005;68(5):2298-303.

[20] Trevisan A, Giuliani A, Scapellato ML, Anticoli S, Carsetti R, Zaffina S, et al. Sex disparity in response to hepatitis B vaccine related to the age of vaccination. Int J Environ Res Public Health 2020;17(1).

[21] Van Damme P. Long-term protection after hepatitis B vaccine. J Infect Dis 2016;214(1):1-3.

[22] Garcia-Agudo R, Aoufi Rabih S, Araque Torres P, Dolores Fraga Fuentes M, Carlos Valenzuela Gamez J, Mancha Ramos J, et al. Efficacy of a hepatitis B vaccination schedule with two cycles of four double doses of conventional vaccine and four doses of adjuvanted vaccine in chronic kidney disease patients evaluated for renal transplantation. Transplant Proc 2012;44 (9):2532-4.

[23] Jacques P, Moens G, Desombere I, Dewijngaert J, Leroux-Roels G, Wettendorff $\mathrm{M}$, et al. The immunogenicity and reactogenicity profile of a candidate hepatitis B vaccine in an adult vaccine non-responder population. Vaccine 2002;20(31-32):3644-9.

[24] Walayat S, Ahmed Z, Martin D, Puli S, Cashman M, Dhillon S. Recent advances in vaccination of non-responders to standard dose hepatitis B virus vaccine. World J Hepatol 2015;7(24):2503-9.

[25] Komatsu H, Klenerman P, Thimme R. Discordance of hepatitis B vaccination policies for healthcare workers between the United States, the United Kingdom and Germany. Hepatol Res 2019.

[26] Boland G, Beran J, Lievens M, Sasadeusz J, Dentico P, Nothdurft H, et al. Safety and immunogenicity profile of an experimental hepatitis B vaccine adjuvanted with AS04. Vaccine 2004;23(3):316-20.

[27] Kundi M. New hepatitis B vaccine formulated with an improved adjuvant system. Expert Rev Vaccines. 2007; 6(2):133-40.

[28] Beran J. Safety and immunogenicity of a new hepatitis B vaccine for the protection of patients with renal insufficiency including pre-haemodialysis and haemodialysis patients. Expert Opin Biol Ther 2008;8(2):235-47.

[29] Raven SFH, Hoebe C, Vossen A, Visser LG, Hautvast JLA, Roukens AHE, et al. Serological response to three alternative series of hepatitis B revaccination (Fendrix, Twinrix, and HBVaxPro-40) in healthy non-responders: a multicentre, open-label, randomised, controlled, superiority trial. Lancet Infect Dis 2020;20(1):92-101.

[30] Machiels JD, Braam EE, van Bentum P, van Vugt M, de Vries-Sluijs T, Schouten I, et al. Vaccination with Fendrix of prior nonresponding patients with HIV has a high success rate. Aids 2019;33(3):503-7.

[31] Lindemann M, Zaslavskaya M, Fiedler M, Wilde B, Heinemann FM, Heinold A, et al. Humoral and cellular responses to a single dose of fendrix in renal transplant recipients with non-response to previous hepatitis B vaccination. Scand J Immunol 2017;85(1):51-7.

[32] Hoebe CJ, Vermeiren AP, Dukers-Muijrers NH. Revaccination with Fendrix(R) or HBVaxPro(R) results in better response rates than does revaccination with three doses of Engerix-B(R) in previous non-responders. Vaccine 2012;30 (48):6734-7.

[33] Barril G, Teruel JL. [Vaccination scheme in advanced chronic kidney disease]. Nefrologia. 2008; 28 (Suppl 3):95-9.

[34] de Silva TI, Green ST, Cole J, Stone BJ, Dockrell DH, Vedio AB. Successful use of Fendrix in HIV-infected non-responders to standard hepatitis B vaccines. J Infect 2014;68(4):397-9.

[35] Fernandez-Prada M, Rodriguez-Fonseca OD, Brandy-Garcia AM, AlonsoPenanes P, Huerta-Gonzalez I, Fernandez-Noval F. Use of hepatitis B AS04C adjuvanted vaccine in HIV patients. Rev Esp Quimioter 2018;31(2):105-9.

[36] Hoerger TJ, Bradley C, Schillie SF, Reilly M, Murphy TV. Cost-effectiveness of ensuring hepatitis B protection for previously vaccinated healthcare personnel. Infect Control Hosp Epidemiol 2014;35(7):845-54.

[37] O’Malley EM, Scott 2nd RD, Gayle J, Dekutoski J, Foltzer M, Lundstrom TS, et al. Costs of management of occupational exposures to blood and body fluids. Infect Control Hosp Epidemiol 2007;28(7):774-82.

[38] Lok AS, McMahon BJ. Practice Guidelines Committee AAftSoLD. Chronic hepatitis B: update of recommendations. Hepatology 2004;39(3):857-61.

[39] Varghese GM, Abraham OC, Mathai D. Post-exposure prophylaxis for blood borne viral infections in healthcare workers. Postgrad Med J 2003;79 (932):324-8.

[40] Solano VM, Hernandez MJ, Montes FJ, Arribas JL. Update of the cost of needlestick injuries in hospital healthcare personnel. Gac Sanit 2005;19 (1):29-35.

[41] Van Der Meeren O, Crasta P, Cheuvart B, De Ridder M. Characterization of an age-response relationship to GSK's recombinant hepatitis B vaccine in healthy adults: An integrated analysis. Hum Vaccin Immunother 2015;11(7):1726-9.

[42] Raheja D, Davila EP, Johnson ET, Deovic R, Paine M, Rouphael N. Willingness to participate in vaccine-related clinical trials among older adults. Int J Environ Res Public Health 2018;15(8).

[43] Flores Muñoz P, Muñoz Escobar, Sánchez Acalo T. Estudio de potencia de pruebas de normalidad usando distribuciones desconocidas con distintos niveles de no normalidad. Study of the power of test for normality using unknown distributions with different levels of non normality. Perfiles 2019; 1 (21):7.

[44] Trevisan A, Frasson C, De Nuzzo D, Nicolli A, Scapellato ML. Significance of anti-HB levels below $10 \mathrm{IU} / \mathrm{L}$ after vaccination against hepatitis B in infancy or adolescence: an update in relation to sex. Hum Vaccin Immunother 2019:1-5.

[45] Van Der Meeren O, Peterson JT, Dionne M, Beasley R, Ebeling PR, Ferguson M, et al. Prospective clinical trial of hepatitis B vaccination in adults with and without type-2 diabetes mellitus. Hum Vaccin Immunother 2016;12 (8):2197-203. 